Anastasia Koroleva*, Andrea Deiwick, Alexander Nguyen, Roger Narayan, Anastasia Shpichka, Olga Kufelt, Roman Kiyan, Victor Bagratashvili, Peter Timashev, Thomas Scheper and Boris Chichkov

\title{
Hydrogel-based microfluidics for vascular tissue engineering
}

DOI 10.1515/bnm-2015-0026

Received December 23, 2015; accepted March 15, 2016; previously published online April 18, 2016

Abstract: In this work, we have explored 3-D co-culture of vasculogenic cells within a synthetically modified fibrin hydrogel. Fibrinogen was covalently linked with PEGNHS in order to improve its degradability resistance and physico-optical properties. We have studied influences of the degree of protein PEGylation and the concentration of enzyme thrombin used for the gel preparation on cellular responses. Scanning electron microscopy analysis of prepared gels revealed that the degree of PEGylation and the concentration of thrombin strongly influenced microstructural characteristics of the protein hydrogel. Human umbilical vein endothelial cells (HUVECs) and human adipose-derived stem cells (hASCs), used as vasculogenic co-culture, could grow in 5:1 PEGylated fibrin gels prepared using 1:0.2 protein to thrombin ratio. This gel formulation supported hASCs and HUVECs spreading and the formation of cell extensions and cell-to-cell contacts. Expression of specific ECM proteins and vasculogenic process inherent cellular enzymatic activity were investigated by immunofluorescent staining, gelatin zymography, western blot and

\footnotetext{
*Corresponding author: Anastasia Koroleva, Nanotechnology Department, Laser Zentrum Hannover e.V, Hollerithallee 8, 30419 Hannover, Germany Phone: +495112788514 ,

E-mail: a.koroleva@lzh.de

Andrea Deiwick, Olga Kufelt, Roman Kiyan and Boris Chichkov: Nanotechnology Department, Laser Zentrum Hannover e.V, Hannover, Germany

Alexander Nguyen: Nanotechnology Department, Laser Zentrum Hannover e.V, Hannover, Germany; and Joint Department of Biomedical Engineering, North Carolina State University, Raleigh, NC, USA Roger Narayan: Joint Department of Biomedical Engineering, North Carolina State University, Raleigh, NC, USA

Anastasia Shpichka: Department of General and Clinical Pharmacology, Penza State University, Penza, Russia Victor Bagratashvili and Peter Timashev: Institute of Photonic Technologies, Research Center of Crystallography and Photonics RAS, Troitsk, Moscow, Russia

Thomas Scheper: Institute of Technical Chemistry, Leibniz Universität Hannover, Hannover, Germany
}

RT-PCR analysis. After evaluation of the optimal gel composition and PEGylation ratio, the hydrogel was utilized for investigation of vascular tube formation within a perfusable microfluidic system. The morphological development of this co-culture within a perfused hydrogel over 12 days led to the formation of interconnected HUVEC-hASC network. The demonstrated PEGylated fibrin microfluidic approach can be used for incorporating other cell types, thus representing a unique experimental platform for basic vascular tissue engineering and drug screening applications.

Keywords: microfluidic hydrogels; PEGylated fibrin; tissue engineering; vasculogenesis.

\section{Introduction}

Engineering of functional blood vessels represents a major challenge in soft tissue reconstruction. The absence of a functional vasculature is the cause of numerous pathologies and is also a major limiting factor in the development of successful tissue engineering therapies. There are two explicit mechanisms of blood vessel growth: vasculogenesis, the de novo formation of blood vessels by endothelial progenitor cells, and angiogenesis, the sprouting of new vessels from preexisting ones. For engineering of microvasculature within a 3-D cell laden biomaterial construct, many biological extracellular matrix (ECM) materials and cell co-cultures have been studied with respect to their vasculogenic potential [1-4]. Mimicking the embryonic environment for vasculogenesis is a popular strategy; for example, experiments on encapsulation of vascular progenitor cells within 3-D biological ECM have been undertaken, in which angioblasts and mesenchymal stem cells self-organize into a microvascular network and form a capillary bed [5]. The ability to replicate a microcapillary network formation by applying adult endothelial cells signifies a useful and feasible option from which clinically relevant vascularized tissue constructs can be further developed.

To mimic native 3-D alignment of living cells in a living tissue, biomaterials should fulfill three main 
objectives: ensure mass transport within the bulk of material, exhibit an appropriate physico-chemical environment, and offer biological sites for integrin-dependent cell adhesion. For several decades, ECM from natural sources has served as an important tool for biologists. ECM materials include purified type I collagen, fibronectin, laminin and reconstituted basement membranes (e.g. Matrigel $\left.{ }^{\circledR}\right)$. These components of the ECM are essential for the establishment of proper cell growth conditions in a 3-D environment by providing adhesion-promoting compounds, as well as, by directing phenotypic cell alignment [6]. However, 3-D culture systems based only on pure ECM components exhibit certain limitations. For example, Matrige $\mathrm{l}^{\circledR}$ contains a population of uncharacterized and potentially tumor inducing growth factors, the concentration of which substantially varies among different batches of this biomaterial [7, 8]. Even after extracting these growth factors, as in purified collagen, modulation of the mechanical properties of collagen is impossible without altering its fiber network density and porosity. Finally, the major limitation of natural ECM systems is their relatively rapid in vitro degradation.

Fibrin has been qualified as an appropriate natural protein matrix material for vascular tissue engineering due to its unique properties [9]. Fibrinogen, which is the fibrin precursor, is a blood protein and can be autologously derived. The physical properties of fibrin can be controlled in many ways. For example, the fibrinogen concentration, fibrinogen to thrombin ratio, $\mathrm{Ca}^{2+}$ concentration, addition/ absence of Factor XIII, and $\mathrm{pH}$ conditions strongly regulate the network density of the fibrin gel [10]. However, fibrin gels undergo degradation and strong matrix remodeling in vitro, which results in construct shrinkage and weight loss [11]. PEGylation of fibrinogen is one possible method to control the degradation characteristics, among other physical properties, and to obtain new possibilities for prolonged 3-D vascular morphogenesis in vitro.

Synthetic and semi-synthetic hydrogels have become popular for engineering of soft tissues and for 3-D cell encapsulation due to their tunable physico-chemical properties and their ability to facilitate transport of oxygen and nutrients by diffusion. Among synthetic hydrogels, poly(ethylene glycol) (PEG) is a commonly used material for tissue engineering applications. PEG offers a mechanically stable, biocompatible 3-D platform in which living cells can be encapsulated and cultured in vitro or implanted in vivo [12]. Many protocols exist for the modification of PEGs with different chemical functional groups; for example, PEG can be conjugated with peptides, enzymes, proteins, and other biomaterials for use in tissue engineering and drug delivery. Conjugation of
ECM-like proteins with PEG can combat the issue of rapid in vitro degradation. PEGylation, as it is called, involves the reaction of hydroxyl terminal groups of a PEG molecule with protein amino groups to yield intra- or inter-molecular cross linking. These crosslinks modify the degradation properties, enhancing their stability and improving their therapeutic potential, particularly for tissue engineering applications.

Several publications have reported on entrapment of living cells within 3-D PEG modified fibrin matrices for studying cell migration, proliferation, differentiation, and tubulogenesis [13-16]. Zhang et al. have reported on the development of a 3-D PEGylated fibrin matrix with tunable physical and mechanical properties [15]. The effects of various formulations of PEGylated fibrin environments on the differentiation of human mesenchymal stem cells (hMSCs) towards a vascular phenotype were investigated by analysis of angiogenic gene expression and phenotypic immunofluorescent staining. hMSCs formed extended multicellular tubes with a clear lumen only when cultured in fibrin matrices modified with difunctional benzoyltriazole carbonate (BTC)-PEG. Galler et al. have examined PEGylated fibrin gel for encapsulation of different toothderived stem cells as a delivery system for cell-based regeneration of dental structures [14]. They have demonstrated that cells loaded into PEGylated fibrin can proliferate and produce collagen, deposit minerals, and increase the expression of mineralization markers after osteogenic induction.

In this work, we describe our studies on the application of PEGylated fibrin gels for fabrication of a hydrogel-based microfluidic chamber. Human umbilical vein endothelial cells (HUVECs) were used as vascular precursor cells and human adipose derived stem cells (hASCs) were used as stromal cells to support HUVEC morphogenesis via secretion of pro-angiogenic growth factors $[2,4]$. It is hypothesized (a) that a cell friendly environment will support in vitro vasculogenesis that is induced by the interaction of HUVECs and hASCs, and (b) that PEGylated fibrin gel can be a strong candidate material for cell encapsulation and hydrogel based microfluidic device fabrication. Following this idea, fibrinogen was PEGylated in different molar ratios and used for the preparation of cell-laden fibrin gels by applying two different thrombin concentrations. The optimal gel properties for cell growth were first investigated via rheological analysis, scanning electron microscopy (SEM) analysis of fibrilar gel network and observations of morphological development of HUVEC-hASCs co-culture. The selected formulations were used in the fabrication of perfusable microfluidic construct emulating vasculature. 


\section{Materials and methods}

\author{
Fibrinogen PEGylation
}

O,O'-Bis[2-( $N$-Succinimidyl-succinylamino)ethyl]poly(ethylene glycol) (PEG-NHS), lyophilized bovine fibrinogen, and bovine thrombin were purchased from a commercial source (Sigma-Aldrich, St. Louis, MO, USA). Bovine fibrinogen was dissolved in sterile PBS at a $25 \mathrm{mg} / \mathrm{mL}$ protein concentration. Thrombin stock solution was prepared at a $100 \mathrm{U} / \mathrm{mL}$ concentration in sterile PBS. Both solutions were stored at $-20^{\circ} \mathrm{C}$ until usage. Prior to protein PEGylation, a PEG-NHS solution was prepared by dissolving PEG-NHS in PBS at a $1.5 \mathrm{mg} / \mathrm{mL}$ concentration. PEGylation was carried out for $2 \mathrm{~h}$ at $37^{\circ} \mathrm{C}$ by addition of PEG-NHS solution to fibrinogen in two different molar ratios, 10:1 and 5:1 PEG-NHS to fibrinogen.

\section{FT-IR analysis of PEGylated fibrinogen}

FT-IR spectra were collected using a Perkin Elmer FT-IR 100 spectrometer. PEGylated and non-modified fibrinogen solutions were lyophilized prior to FT-IR sample preparation. All of the samples were prepared as potassium bromide $(\mathrm{KBr})$ pellets, which were measured against pure $\mathrm{KBr}$ as a blank.

\section{Characterization of fibrin and PEGylated fibrin networks by SEM}

For the evaluation of PEGylated fibrin network architechture, the different gel formulations were fixed with $4 \%$ paraformaldehyde and dehydrated with a sequence of serially diluted ethanol (30\%-100\%). The gels were then dried using a critical point dryer and subsequently sputter coated with $100 \mathrm{~nm}$ of gold. SEM imaging was performed with a Quanta 400F microscope (FEI Company, OR, USA).

\section{Rheological characterization of PEGylated fibrin gels}

To study the viscoelastic properties of PEGylated and non-modified fibrin gels, dynamic shear oscillation experiments were carried out using a rheometer (Physica MCR 301, Anton Paar Germany GmbH). Cell-free gel samples were prepared from $1 \mathrm{~mL}$ of the fibrinogen and thrombin mixtures in a $25 \mathrm{~mm}$ diameter cylindrical mold and transferred to the bottom plate of the rheometer. The upper plate was then lowered to a measuring gap size of $1.7 \mathrm{~mm}$. The viscoelastic measurements of the gels were performed using an amplitude sweep from $0.01 \%$ to $100 \%$ deformation. The operating temperature was kept constant at $22 \pm 0.1{ }^{\circ} \mathrm{C}$. To determine the stiffness of the materials, the complex shear modulus $\left(\mathrm{G}^{*}\right)$ was calculated according to equation (1):

$$
\left(G^{*}\right)=\sqrt{\left(G^{\prime}\right)^{2}+}\left(G^{\prime \prime}\right)^{2},
$$

where $\left(G^{\prime}\right)$ is the storage modulus and $\left(G^{\prime \prime}\right)$ is the loss modulus. The results are given as average of four independently prepared gel samples from each formulation.

\section{Cell culture}

hASCs were obtained from human adipose tissue that was collected from patients undergoing elective plastic surgery after obtaining informed consent. Isolation of the cells took place according to a protocol described elsewhere [17]. HUVECs were purchased from Lonza (Basel, Switzerland). All of the cell types used in this study, including hASCs, were maintained in EBM-2 medium (Lonza, Basel, Switzerland) supplied with $1 \%$ fetal bovine serum (FBS) and $1 \%$ penicillin-streptomycin and used in the experiment at passages 4-6 for endothelial cells and 5-7 for ASCs.

\section{Encapsulation of cells into PEGylated fibrin}

Constructs consisting of a target containing $5 \times 10^{4}$ cells per gel (4:1 ratio of HUVECs to ASCs) with a $25 \mathrm{mg} / \mathrm{mL}$ fibrinogen concentration were formed by first diluting $20 \mu \mathrm{L}$ of cell suspension with $30 \mu \mathrm{L}$ of a PEGylated fibrinogen solution. After ensuring thorough mixing, a sufficient volume of thrombin stock solution to form a fibrinogen to thrombin ratio of either $1 \mathrm{mg}$ to $1 \mathrm{U}$ or $1 \mathrm{mg}$ to $0.2 \mathrm{U}$ was added. The mixture was immediately cast within a 24 well plate and cultured for 14 days with semi-weekly media changes. The six gel formulations consisting of unmodified fibrinogen, 5:1 PEGylated fibrinogen, or 10:1 PEGylated fibrinogen with either $1 \mathrm{U}$ of thrombin per $1 \mathrm{mg}$ protein or $0.2 \mathrm{U}$ per $1 \mathrm{mg}$ were utilized for studying cellular response.

\section{Fabrication of perfusable PEGylated fibrin microfluidic device}

The chamber for perfused cell-laden hydrogel culture was fabricated from polydimethylsiloxane (PDMS) (Sylgard 184, Dow Corning, Midland, MI, USA). The cavity for casting hydrogel, which was $6 \times 6 \times 40 \mathrm{~mm}$ in depth, width and length, was produced by casting the resin around a polyoxymethylene piece. Silicone tubes (Watson Marlow Limited, Falmouth Cornwall, England) with an inner diameter of $0.6 \mathrm{~mm}$ were connected on opposite sides of the chamber using additional PDMS as an adhesive. An approximately $3 \mathrm{~mm}$ thick flat PDMS sheet, in which a single $6 \mathrm{~mm}$ hole was removed from the center with a coring tool, was used to enclose the chamber; the hole was utilized later to fill the chamber with the hydrogel mixture. The microfluidic channel within the hydrogel was formed by threading a $0.5 \mathrm{~mm}$ thick glass optical fiber coated with bovine gelatin, which was used as a release agent, through the two silicone tubes. The prepared chamber was kept overnight in sterile conditions for PDMS solidification.

A PEGylated fibrin gel containing hASCs and HUVECs was prepared as follows: $300 \mu \mathrm{L}$ of cell suspension containing $3 \times 10^{6}$ HUVECs and $7.5 \times 10^{5} \mathrm{hASC}$ was distributed equally between $650 \mu \mathrm{L}$ of PEGylated fibrinogen $(25 \mathrm{mg} / \mathrm{mL}$ 5:1 ratio) and $650 \mu \mathrm{L}$ of thrombin $(10 \mathrm{U} / \mathrm{mL})$ solutions. These cell-containing gel precursor solutions were filled in two separate $2 \mathrm{~mL}$ syringes and connected with a mixing nozzle. Fixation of syringes in this manner allowed synchronized injection and thorough mixing of PEGylated fibrin components within the chamber cavity. The injection was carried out via the $6 \mathrm{~mm}$ wide chamber opening, which afterwards was sealed with fitted PDMS cork and insulated with additional liquid PDMS. 
After $1 \mathrm{~h}$ of gelation at $37^{\circ} \mathrm{C}$, the glass optical fiber was slowly withdrawn from the gel via the silicone tubes, resulting in a $1 \mathrm{~mm}$ thick channel within the cell-laden PEGylated fibrin. The perfusion of gel construct with EBM-2 cell culture media was conducted via the silicone tubes using a multichannel peristaltic pump (Watson Marlow, Falmouth, Cornwall, UK). The gel chamber, cell culture media reservoir, and tubes were kept in an incubator at $37^{\circ} \mathrm{C}$ in a humidified atmosphere containing $95 \%$ air and $5 \% \mathrm{CO}_{2}$ and perfused at a $1 \mathrm{~mL} /$ min flow rate.

\section{Fluorescent cell staining}

HUVECs and hASCs were labeled with Vybrant ${ }^{\circledR}$ DiO and with Vybrant ${ }^{\circledR}$ Dil cell tracers (Life Technologies, Darmstadt, Germany) according to the manufacturer's instructions. Immunofluorescence staining was performed by a two-step indirect method. Briefly, the gels were fixed in 4\% paraformaldehyde for $1 \mathrm{~h}$ and permeabilized with $1 \%$ Triton X-100/PBS. To prevent nonspecific antibody binding, a $2 \%$ bovine serum albumin/PBS solution was applied at $37^{\circ} \mathrm{C}$ for 2 h. After blocking, the gels were incubated with primary antibodies (rabbit anti PECAM/CD31 1:50, Abcam, Cambridge, UK; mouse anti alpha smooth muscle actin (aSMA), 1:1000, Sigma-Aldrich, Deisenhofen, Germany; rabbit anti MMP14,1:500, Abcam; mouse anti collagen type IV, 1:50, Progen, Heidelberg, Germany; mouse anti laminin, 1:250, Sigma-Aldrich) diluted in $1 \%$ Triton X-100/PBS overnight at 4 ${ }^{\circ} \mathrm{C}$. After several washing steps, the gels were incubated with a fluorescence-conjugated secondary antibody at an appropriate dilution (Alexa Fluor 488 conjugated Goat anti-rabbit IgG (H+L), Dianova, Hamburg, Germany/Alexa Fluor ${ }^{\circledR} 555$ conjugated Goat anti-mouse IgG (H+L), Life Technologies) for $1 \mathrm{~h}$ at $37{ }^{\circ} \mathrm{C}$. Cell nuclei were stained with Hoechst 33342. Gel samples were washed three times and stored in PBS prior to confocal microscopy (Inverted Confocal LSM Zeiss 780).

\section{Western blot analysis}

Total protein was isolated from cell monolayers and gel cultures with ice-cold RIPA-lysis buffer (25 mM Tris-HCl (pH 7.6), $150 \mathrm{mM} \mathrm{NaCl,} \mathrm{1 \%}$ Nonidet P-40, $0.1 \%$ SDS, and $1 \%$ sodium deoxycholate) by sonication. The total protein concentration of the resulting lysate was quantified using a BCA protein assay (Roth, Karlsruhe, Germany). Equivalent amounts of total protein $(10 \mu \mathrm{g})$ were loaded on a $10 \%$ polyacrylamide resolving gel and separated by SDS-PAGE under reducing conditions. After electrophoresis, proteins were transferred to a polyvinylidene fluoride (PVDF) membrane (Roth) by semi-dry blotting. The membrane was blocked for $1 \mathrm{~h}$ in $1 \times$ Roti $^{\circledast}$ Block (Roth) at room temperature. The membrane was then probed with a rabbit anti-human MT1-MMP antibody (MMP14 hinge region (1:1000, Abcam) overnight at $4{ }^{\circ} \mathrm{C}$. After washing, the membrane was incubated with a horseradish peroxidase-conjugated secondary antibody for $1 \mathrm{~h}$ at room temperature. Detection of the secondary antibody was performed with an enhanced chemiluminescence system using the Pierce ECL substrate (Thermo FisherScientific, Schwerte, Germany). In addition, the membrane was reprobed with a mouse anti $\beta$-actin antibody (1:50,000; Sigma-Aldrich) for an internal loading control.
The relative band intensities in the scanned blots were analyzed by densitometry with Image J software (National Institutes of Health, MD, USA).

\section{Gelatin zymography}

Gelatin zymography was performed on $10 \%$ polyacrylamide gels copolymerized with $1 \mathrm{mg} / \mathrm{mL}$ gelatin from porcine skin (SigmaAldrich). Equal amounts of protein $(10 \mu \mathrm{g})$ were diluted in sodium dodecyl sulphate (SDS) sample buffer (final concentration: $50 \mathrm{mM}$ Tris-HCl pH 6.8; 1\% (w/v) SDS; 10\% (v/v) glycerol; 0.025\% (w/v) bromophenol blue) under non-reducing conditions without heating and loaded onto the gel. After electrophoresis, gels were washed twice for $30 \mathrm{~min}$ in $2.5 \%$ Triton $\mathrm{X}-100$ at room temperature and incubated overnight in enzyme buffer (final concentration: $50 \mathrm{mM}$ Tris$\mathrm{HCl}, \mathrm{pH} 7.5 ; 5 \mathrm{mM} \mathrm{CaCl}_{2} ; 100 \mathrm{mM} \mathrm{NaCl} ; 0.1 \% \mathrm{NaN}_{3}$ ) at $37^{\circ} \mathrm{C}$. Gels were stained in Coomassie Brilliant Blue R-250 $(2.5 \mathrm{mg} / \mathrm{mL}$ in 10\% acetic acid and 10\% 2-propanol) for $30 \mathrm{~min}$. Gels were destained in 10\% acetic acid and 10\% 2-propanol. Gelatinolytic activity appears as clear bands against a blue background.

\section{RNA extraction and RT-PCR}

The total RNA was extracted from cells cultured either as a monolayer or within the gels with TRIZOL ${ }^{\circledR}$ Reagent (Invitrogen, Germany), which was used according to the manufacturer's protocol. $2 \mu \mathrm{g}$ DNAse-treated RNA was reverse transcribed to cDNA using MuLV reverse transcriptase (Invitrogen, Germany). Polymerase Chain Reaction (PCR) amplification was performed in a peqSTAR thermocycler (PEQLAB Biotechnologie GMBH, Erlangen, Germany) using gene-specific primers (Unmodified DNA oligonucleotides (salt-free), Eurofins MWG Operon, Ebersberg, Germany) and Taq polymerase (Qiagen, Hilden, Germany). PCR cycles and annealing temperatures were optimized for each set of primers. The various cDNA samples were adjusted to equal input concentrations by the expression level of glyceraldehyde 3-phosphate dehydrogenase (GAPDH). The following sequences were used: forward primer GCTTGCAAGTAACAGGCAAA and reverse primer AAATTCTCCGTGTCCATCCA for MT1-MMP (NM_004995.3), forward primer GCATCCAGACTTCCTCAGGC and reverse primer AGGTATTGCACTGCCAACTC for MMP-2 (NM_004530.4), forward primer CGCAGACATCGTCATCCAGT and reverse primer GGATTGGCCTTGGAAGATGA for MMP-9 (NM_004994.2), forward primer TAAATACCTGTTGCTGGGCCG and reverse primer TCCAGAACTCGTCTTCGTCG for MMP-19 (NM_002429.5), forward primer CGTAAAGCTATTCGCCGTGC and reverse primer AACCACTGGTCCTTGAAAACA for MT3-MMP (NM_005941.4), and forward primer CTGCACCACCAACTGCTT and reverse primer AGCTCAGGGATGACCTTGC for GAPDH (NM_001289746.1). The amplified fragments were $440 \mathrm{bp}$ for MMP-2, 589 bp for MT1- MMP, 406 bp for MMP-9, 650 bp for MT3-MMP, 302 bp for MMP-19, and $219 \mathrm{bp}$ for GAPDH. Amplified PCR products were separated by electrophoresis on FlashGel ${ }^{\circledR}$ DNA Cassettes (Lonza, Basel, Switzerland), photographed with the FlashGel ${ }^{\circledR}$ Camera, and documented by FlashGel ${ }^{\circledR}$ capture software (FlashGel System, Lonza, Basel, Switzerland). 


\section{Results}

\section{FT-IR analysis of fibrinogen PEGylation.}

The PEGylation via NHS-functionalized PEG (PEG-NHS) was verified via FT-IR measurements of the lyophilized gels [18]. Figure 1A shows the corresponding FT-IR spectra of pure fibrinogen, NHS-functionalized PEG, and the gels modified with 5:1 and 10:1 molar ratios of PEG to fibrinogen. The PEG-NHS shows a characteristic triplet band of the succinimidyl ester $\left(1739,1782\right.$, and $\left.1813 \mathrm{~cm}^{-1}\right)$, which is not present in the modified gels due to imide ring-opening within the reaction with primary amino groups $[19,20]$. The modified gels show an increasing band at $1103 \mathrm{~cm}^{-1}$, indicating the insertion of PEG-derived (C-O) units depending on the amount of added NHS-activated PEG. Similar increases were observed for amide I and amide II bands at 1650 and $1532 \mathrm{~cm}^{-1}$ due to the amides present in PEG-NHS. The growth of the characteristic succinimidyl ester peaks and the increasing amide content are indicative of successful modification of fibrinogen.

\section{Rheological characterization of PEGylated fibrin gels}

Storage and loss moduli were determined for 5:1 and 10:1 molar ratios of PEGylated fibrin, as well as, for non-modified fibrin gels using a parallel plate rheometer. Complex shear moduli ( $\left.G^{*}\right)$, which represent the materials' stiffness, were calculated according to the equation (1) (Figure 1B). The 5:1 PEGylation ratio increased the material stiffness by $30 \%$ in comparison to non-modified fibrin (161 vs. $112 \mathrm{~Pa})$. However, an increase in the PEG content in the
A

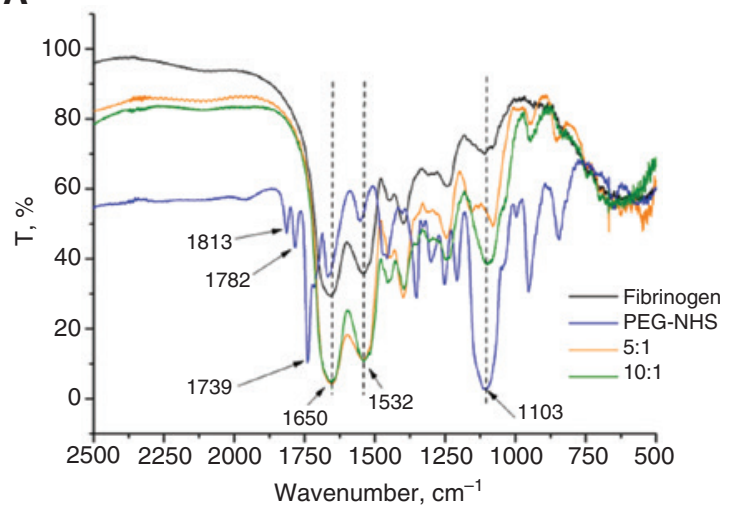

B

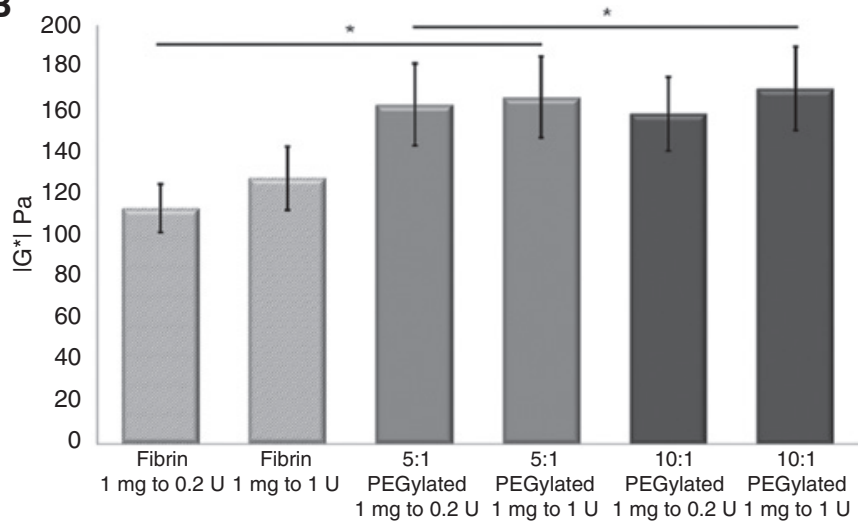

C

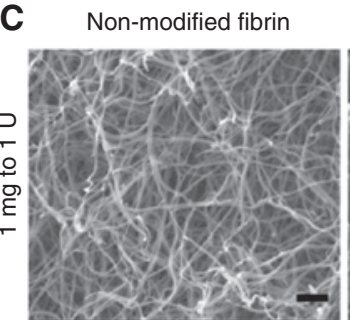

5:1 PEGylated fibrin
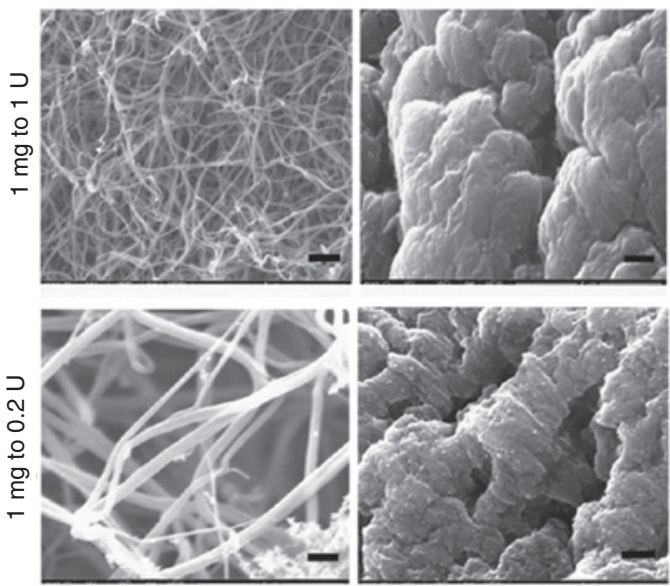

10:1 PEGylated fibrin
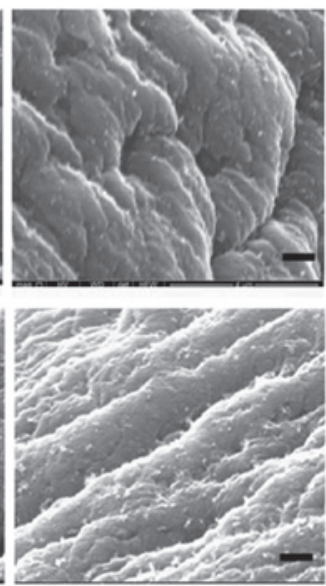

D 5:1 PEGylated fibrin
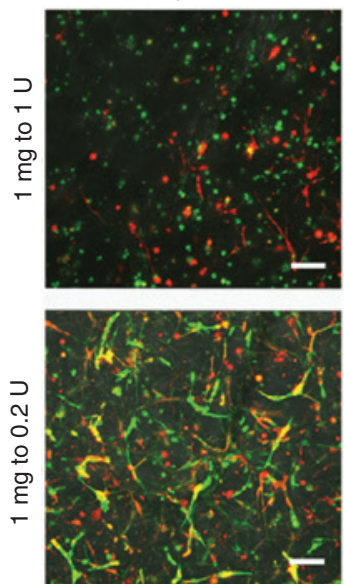

10:1 PEGylated fibrin
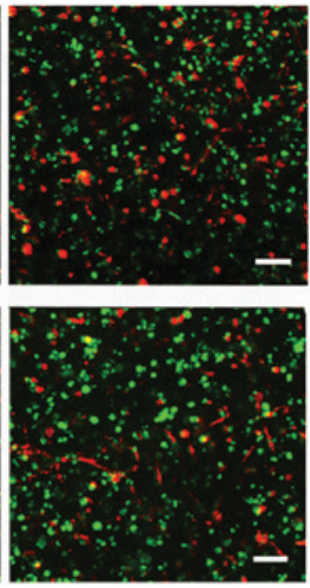

Figure 1: Characterization of PEGylated fibrin vs. non-modified fibrin.

(A) FT-IR spectra of pure fibrinogen, NHS-functionalized PEG (PEG-NHS) and PEGylated fibrinogen with given molar ratios of PEG-NHS to fibrinogen; (B) Complex shear moduli of fibrin hydrogels with various molar ratios of PEG to fibrin and thrombin units (U) used for gel crosslinking, given as absolute values. *indicates significant difference of $\mathrm{p}<0.5$; (C) SEM analysis of PEGylated vs. non-modified fibrin. Scale bars are $1 \mu \mathrm{m}$ long; and (D) Encapsulation of HUVECs (green) and hASCs (red) into PEGylated fibrin. The cells were labeled with DiO and DiL cell trackers before encapsulation and maintained within gels for 7 days. The scale bars are $100 \mu \mathrm{m}$ long. 
hydrogel composition up to 10:1 ratio did not significantly boost its stiffness. The G* moduli of 10:1 PEGylated fibrin were comparable with those of 5:1 PEGylated gels (157 vs. $161 \mathrm{~Pa})$. A slight stiffness difference was observed between the gels prepared using 1 and 0.2 units of thrombin per $\mathrm{mg}$ of fibrinogen. The hydrogels prepared using the lower $0.2 \mathrm{U}$ thrombin concentration had overall 3\%-11\% lower complex shear moduli values in comparison to gels prepared using $1 \mathrm{U}$ thrombin. The PEGylated fibrin hydrogels were observed to be less elastic in comparison to fibrin gels prepared from non-modified fibrinogen and thrombin. The dependence of PEGylation ratio on the stiffness is shown by statistically significant differences $(p<0.05)$ among the PEGylated groups and non-modified fibrin.

\section{SEM of PEGylated fibrin}

SEM revealed distinct differences in fiber morphology among various PEGylated fibrin gels. In comparison to the non-modified fibrin matrix (Figure 1C), the structure of all of the PEGylated hydrogels appears to be strongly modified by the addition of PEG-NHS. The degree of PEGylation as well as the concentration of thrombin used for gel preparation also influenced the density of the protein hydrogel. Gels prepared from 10:1 PEGylated fibrinogen and both thrombin concentrations exhibited very densely packed fiber architectures without pores. 5:1 PEGylation resulted in more flocculent network alignment, with nonuniform pore distribution and pore sizes. The 5:1 PEGylated fibrin gel prepared with less concentrated thrombin (1 mg; $0.2 \mathrm{U}$ ratio) exhibited a more microporous structure and an internal fibrillar network (see Figure 2 middle right image). These results of SEM analysis correlate with our observations of cell behavior, in which growth of HUVECs and hASCs was inhibited by highly modified fibrin gels and increased thrombin concentrations.

\section{Morphological analysis of HUVECs and hASCs cultured within PEGylated fibrin gels}

To evaluate how differently prepared PEGylated fibrin gels influence cell behavior in vitro, hASCs and HUVECs were embedded into PEGylated fibrin gels, followed by cell behavior analysis after 7 days of culture (Figure 1D). The gels with increased stiffness, prepared from 10:1 PEGylated fibrinogen with thrombin concentrations of 1:1
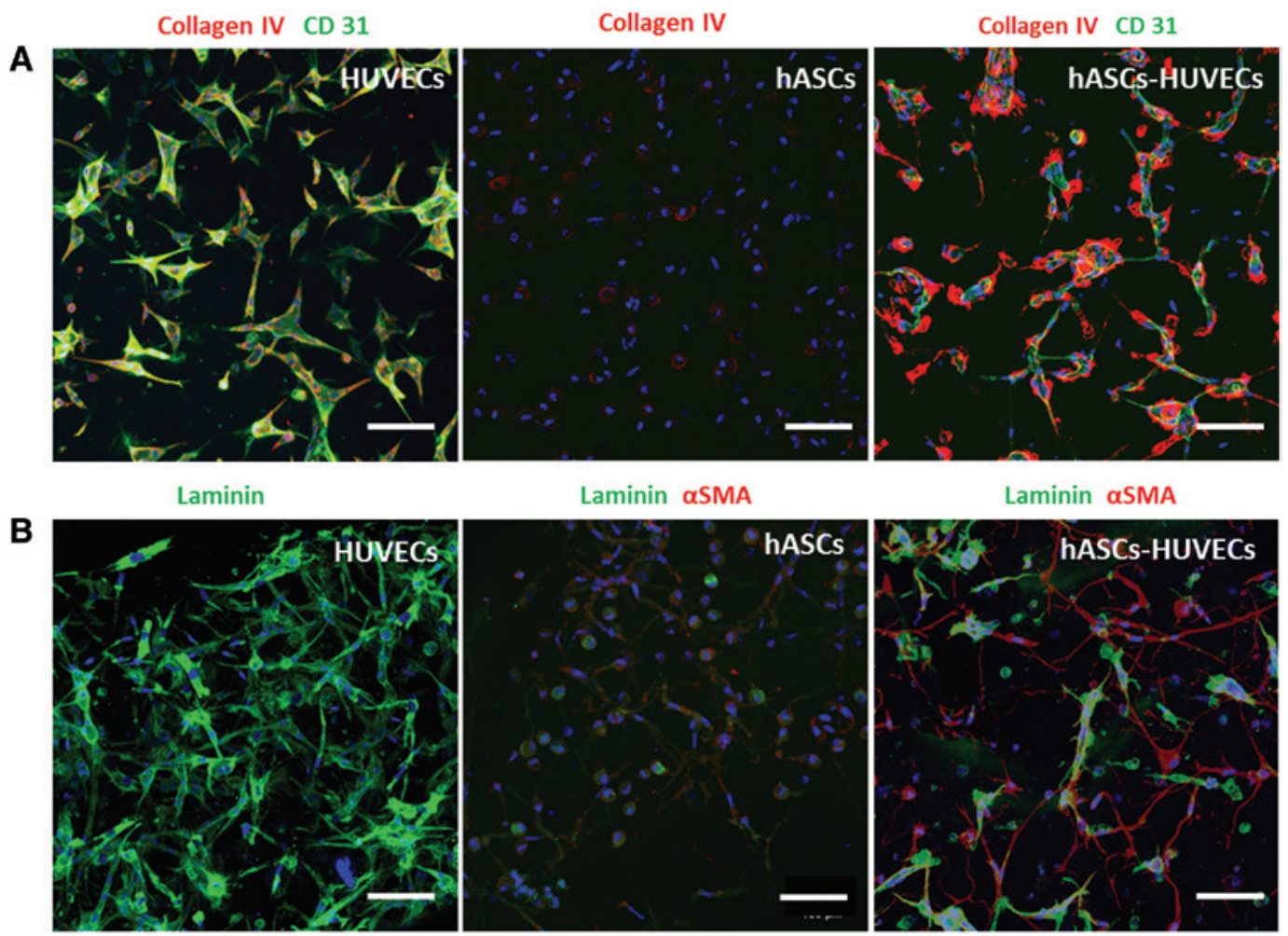

Figure 2: Expression of cellular and ECM proteins in hASC and HUVEC monocultures and HUVEC-hASC co-cultures within 3-D PEGylated fibrin gels.

Scale bars $=100 \mu \mathrm{m}$. 
and 1:0.2, did not support 3-D cell spreading. hASCs and HUVECs cultured within 10:1 PEGylated fibrin gels did not adequately spread within the gel, revealing rounded morphologies and an absence of outgrowth. 3-D gels prepared from 5:1 PEGylated fibrinogen and a 1:1 ratio of protein to thrombin could only partially support spreading and outgrowth of hASCs but not of endothelial cells. In contrast, 5:1 PEGylated fibrin gels with lower stiffness, which were prepared using a 1:0.2 protein to thrombin ratio, supported hASC and HUVEC growth and spreading, with the formation of cell extensions and cell-to-cell contacts (Figure 1D lower left image; yellow color indicates co-localization of hASCs and HUVECs). Therefore, 5:1 PEGylation ratio and $0.2 \mathrm{U}$ thrombin ratio were chosen for further cell encapsulation experiments.

\section{HUVECs and hASCs express various MMPs and MT-MMPs when cultured in PEGylated fibrin gels}

The influence of PEGylated fibrin on the expression of matrix metalloproteinases (MMP) in HUVEC-hASC vasculogenic co-culture was compared to non-modified fibrin and to a traditional 2-D culture in terms of its enzymatic activity. Endothelial cells have been described to produce several MMP such as MMP-2, MMP-9, and MMP-19 as well as the membrane-type matrix metalloproteinases MT1-MMP and MP3-MMP; these molecules are implicated in regulation of angiogenesis [21, 22]. The protease membrane type-1 matrix metalloproteinase (MT1-MMP) is involved in the development of endothelial cell sprouts and is required for capillary formation. According to the results of MT1-MMP immunocytochemistry, the cellular expression of MT1-MMP in solo cultures of hASCs and HUVECs in PEGylated fibrin was much lower than the expression in co-culture; the strongest fluorescence signal in Figure 3A occurred in the gel containing both cell types. Western blot analysis of MT1-MMP indicated that its activity after $4 \mathrm{~h}$ post-seeding was enhanced in both PEGylated and non-modified 3-D gels as compared to 2-D culture (Figure 3B). MT1-MMP activity in 3-D PEGylated fibrin gels was markedly increased starting from day 6 (bands 8,9,11,12) and was comparable to the activity in non-modified fibrin.

As demonstrated by gelatin zymography (Figure 4A), pro-MMP-2 was found to be active within 2-D culture, 3-D non-modified, and PEGylated fibrin gels from the beginning of the culture. Considerable expression of the active form of MMP-2 was detected on day 6 . It must be noted that the lytic activity of both latent and active forms of MMP-2 was significantly stronger for cells cultured within 3-D gels compared to those in 2-D culture.

To study the MMPs that are expressed by HUVECshASCs cultured in 3-D PEGylated fibrin, RT-PCR was used to assess the expression and regulation of MMP on a genetic level. Figure 4B shows that cells expressed considerable amounts of MMP-2, MT1-MMP, and MT3-MMP mRNAs in both 2-D and 3-D culture conditions. Compared to the data obtained from the gels $4 \mathrm{~h}$ after seeding, MT1-MMP was constantly upregulated over the period of 21 days for all of the culture conditions. The upregulation
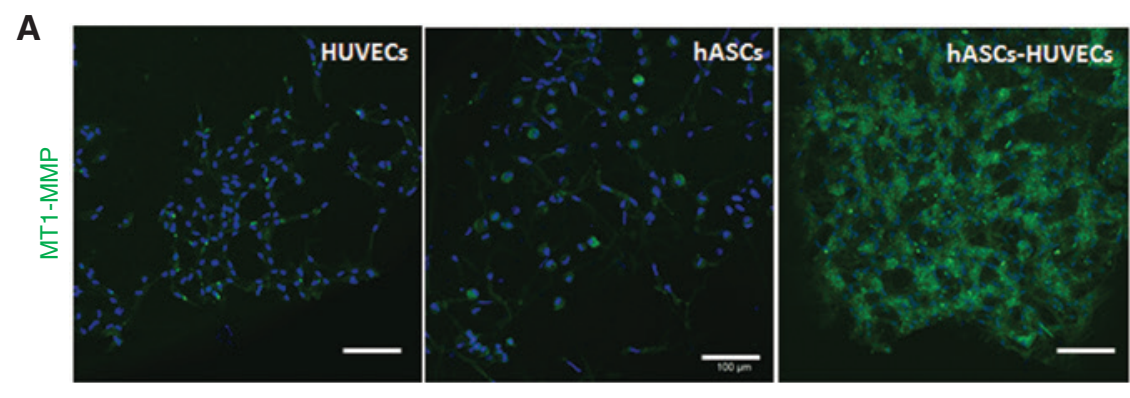

B

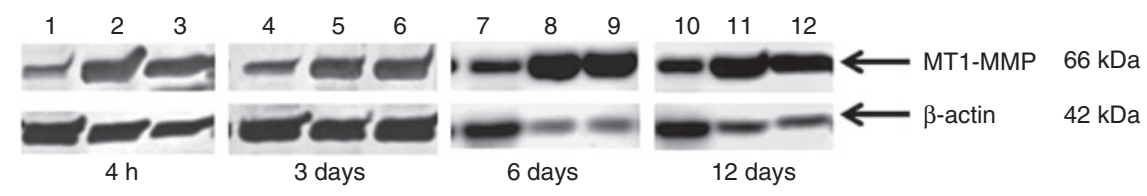

Figure 3: Expression of MT1-MMP within PEGylated fibrin gel.

(A) MT1-MMP immunofluorescent staining of hASC and HUVEC monocultures as well as hASC-HUVEC co-cultures within 3-D PEGylated fibrin gels; MT1-MMP is green, cell nuclei are blue (scale bars=130 $\mu \mathrm{m}$ ); and (B) Western blot analysis of hASC-HUVEC MT1-MMP activity in 2-D (bands 1,4,7,10), 3-D PEGylated (bands 2,5,8,11), and 3-D non-modified fibrin gels (bands 3,6,9,12). 

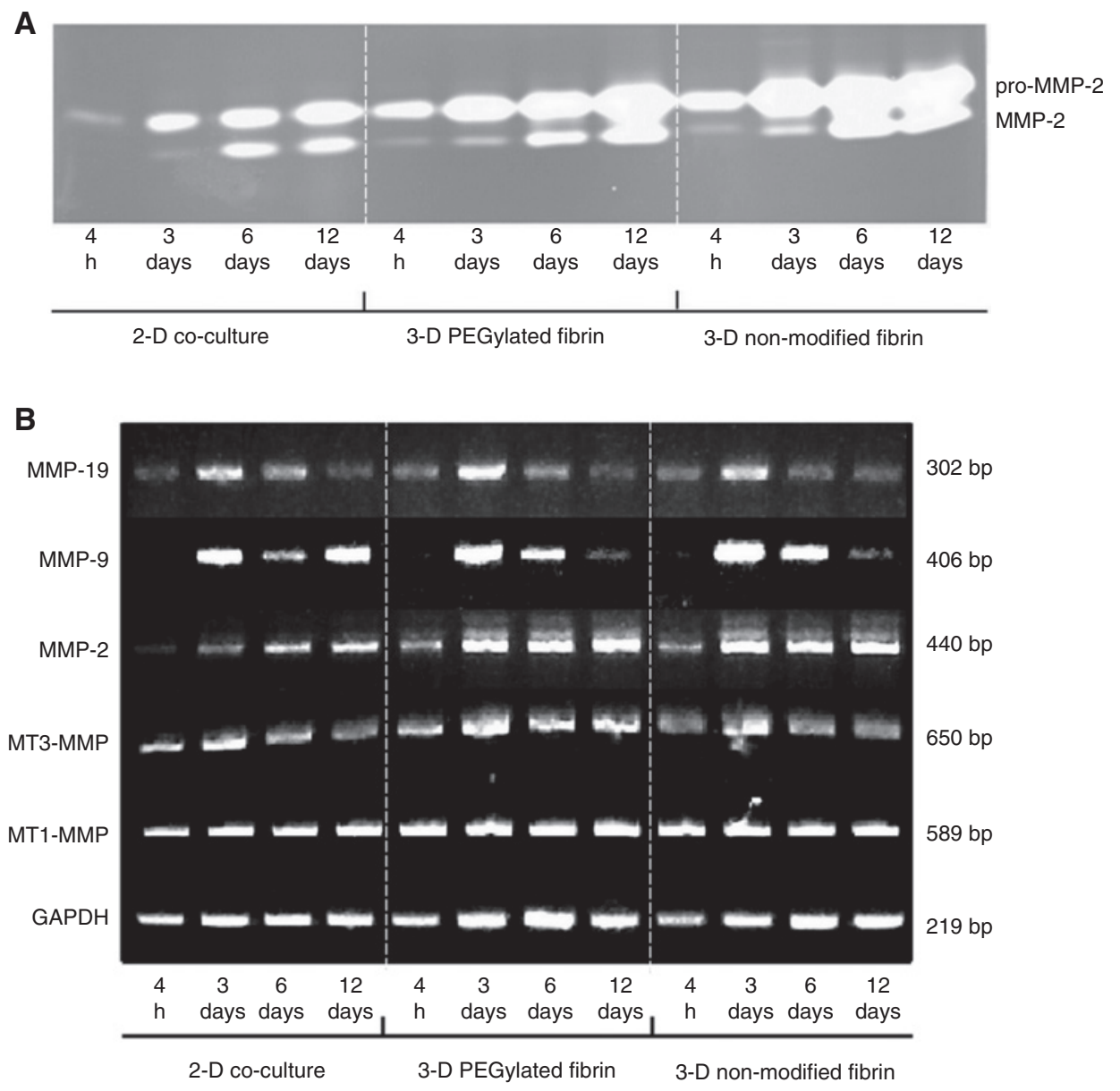

Figure 4: Comparative molecular biological demonstration of cellular MMPs activity at different time points of culture.

(A) zymographic analysis of MMP-2; lytic bands at $72 \mathrm{kDa}$ correspond to latent form of MMP-2 and bands at $62 \mathrm{kDa}$ represent activated form of enzyme; and (B) RT-PCR analysis of HUVEC-hASC MMPs activity in PEGylated fibrin compared with 2-D co-culture and culture in non-modified fibrin.

of MT3-MMP genes was observed starting from day 3. In contrast to the activity of the HUVEC-hASC culture under 2-D conditions and in non-modified fibrin gels, upregulation of MT3-MMP continued over 21 days. MMP-9 and MMP-19 genes were also upregulated starting from day 3; unlike gelatinase MMP-2, these MMPs genes were downregulated to day 21 in both PEGylated and non-modified fibrin 3-D cell co-cultures.

\section{Cellular and ECM protein expression in HUVEC-hASC co-culture within PEGylated fibrin gels}

No significant expression of collagen IV was detected in hASC monoculture within PEGylated fibrin; however, collagen IV expression was present at a low level in HUVEC monoculture. Expression of this protein by HUVECs was significantly increased in co-cultures, especially in regions of HUVEC and hASC contact (Figure 2A). Culture of hASCs alone in PEGylated fibrin gels resulted in relatively low expression of $\alpha \mathrm{SMA}$, with a non-filamentous diffuse distribution (Figure 2B middle). However, coculture of hASCs with HUVECs resulted in a significant increase in $\alpha$ SMA expression and its organization into fibers in hASCs in direct contact with or in close proximity to HUVECs (Figure 2B right). Laminin was present throughout the co-cultures; its highest accumulation was also observed to follow the geometry of HUVECs (Figure 2B right).

\section{Fabrication of hydrogel microfluidic chamber}

Since hydrogels are porous and mechanically weak in general, they cannot withstand the application of high pressure. Therefore, the main concern in creation of a hydrogel-based perfusable system is a proper mechanical 
support of the hydrogel construct. In order to achieve the flow of fluid (media) through the created channel without any leakage, a sealed bioreactor that completely encases the construct in a silicone material was designed. Proper sealing of the bioreactor seams eliminated alternative pathways for fluid flow and constrained the media to the intended path through the fabricated channel. The casting of gel within the chamber was performed by simultaneously injecting the precursor compounds through a mixing nozzle. The efficient mixing of PEGylated fibrin, thrombin, and cell suspension supported consistent distribution of cells within the chamber volume. Cross-linking of fibrin took place within minutes, which additionally prevented settling of the cells by gravity and therefore contributed to the homogeneous 3-D arrangement of HUVECs and hASCs within the chamber. Chamber perfusion was performed $1 \mathrm{~h}$ after casting. The size of the chamber $(6 \times 6 \times 40 \mathrm{~mm}$ $\mathrm{W} \times \mathrm{D} \times \mathrm{L}$ ) was chosen according to our previous perfusion experiments, in which the area supplied with fresh media by a $1 \mathrm{~mm}$ wide channel was established to be of the order of $5 \mathrm{~mm}$. Figure $5 \mathrm{~A}$ shows a cross-section of a $12 \times 12 \times 40 \mathrm{~mm}$ large construct with a $5 \mathrm{~mm}$ middle core supplied by media.

\section{Vasculogenesis in PEGylated fibrin microflu- idic device}

Initial observations showed that cellular responses were related to the ratio of fibrinogen PEGylation and the concentration of thrombin used for cross-linking of the gel. PEGylated fibrin gels with high stiffness affected cell growth and formation of cellular extensions. Gels with lower stiffness gave rise to cell spreading and establishment of cell to cell contacts and interconnections. For the fabrication of a hydrogel perfusable chamber, a 5:1 PEGylated fibrin cross-linked with a 1:0.2 fibrinogen to thrombin ratio was chosen. The hydrogel chambers were continuously perfused for 12 days.

Cellular outgrowths and tubular structure lengths were not affected by the PEGylated fibrin environment. The average length of cellular outgrowths and tubular structures in PEGylated fibrin are comparable with those of natural fibrin at days 3, 6 and 12 (Figure 6a).

A day after cell encapsulation, HUVECs and ASCs displayed rounded morphologies. hASCs spreading was observed to start at day two, whereas HUVECs remained rounded at this time (Figure 6b). Further culture resulted

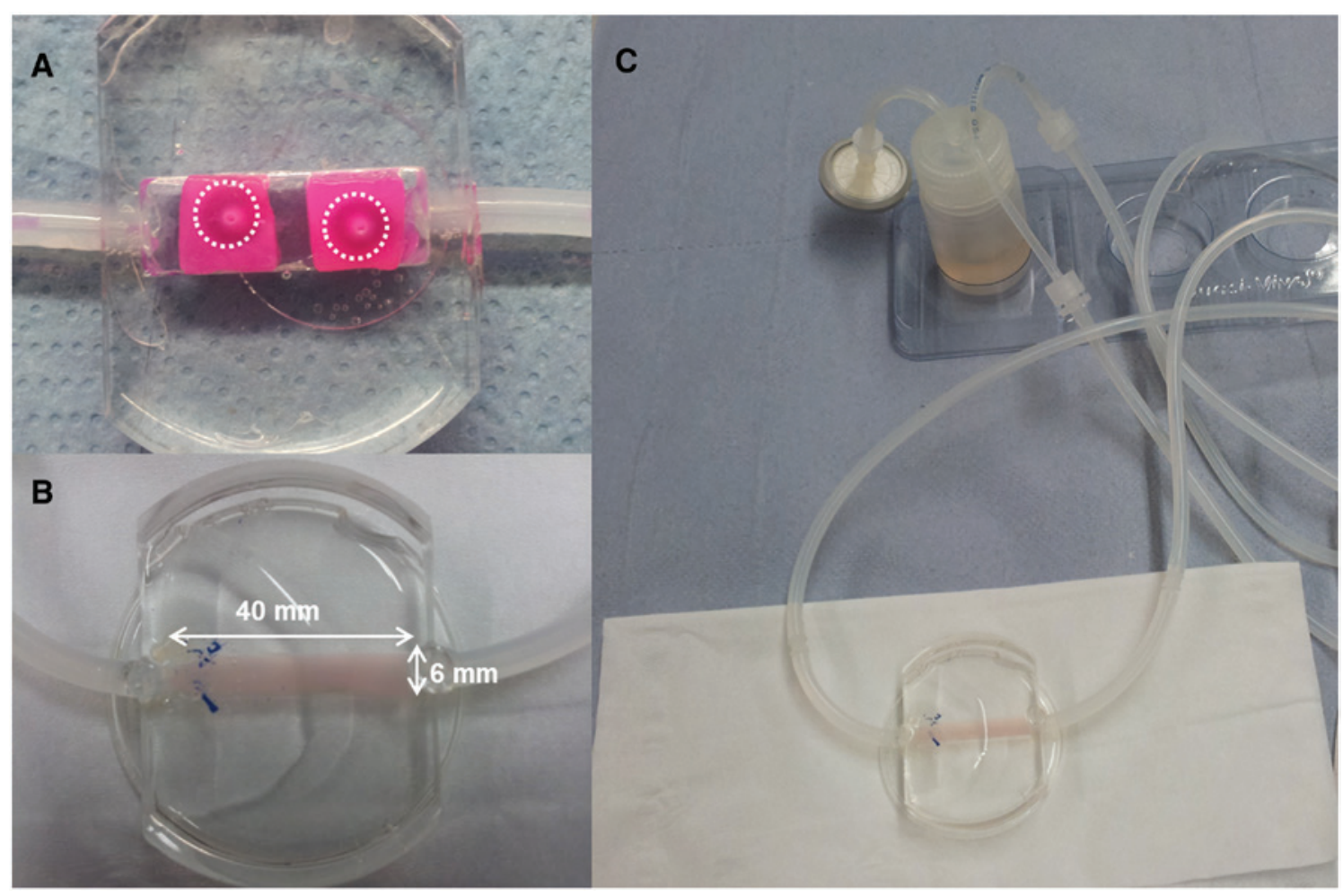

Figure 5: Silicone chamber for insulated hydrogel microfluidics.

(A) Cross section of $12 \times 12 \times 40 \mathrm{~mm}$ construct; white dotted circles indicate the $5 \mathrm{~mm}$ middle core supplied by media; (B) silicone chamber of reduced $6 \times 6 \times 40 \mathrm{~mm}$ size filled with a PEGylated hydrogel containing a co-culture of HUVECs and hASCs after 10 days of perfusion culture; and (C) silicone chamber connected to media reservoir via tubes. 
a
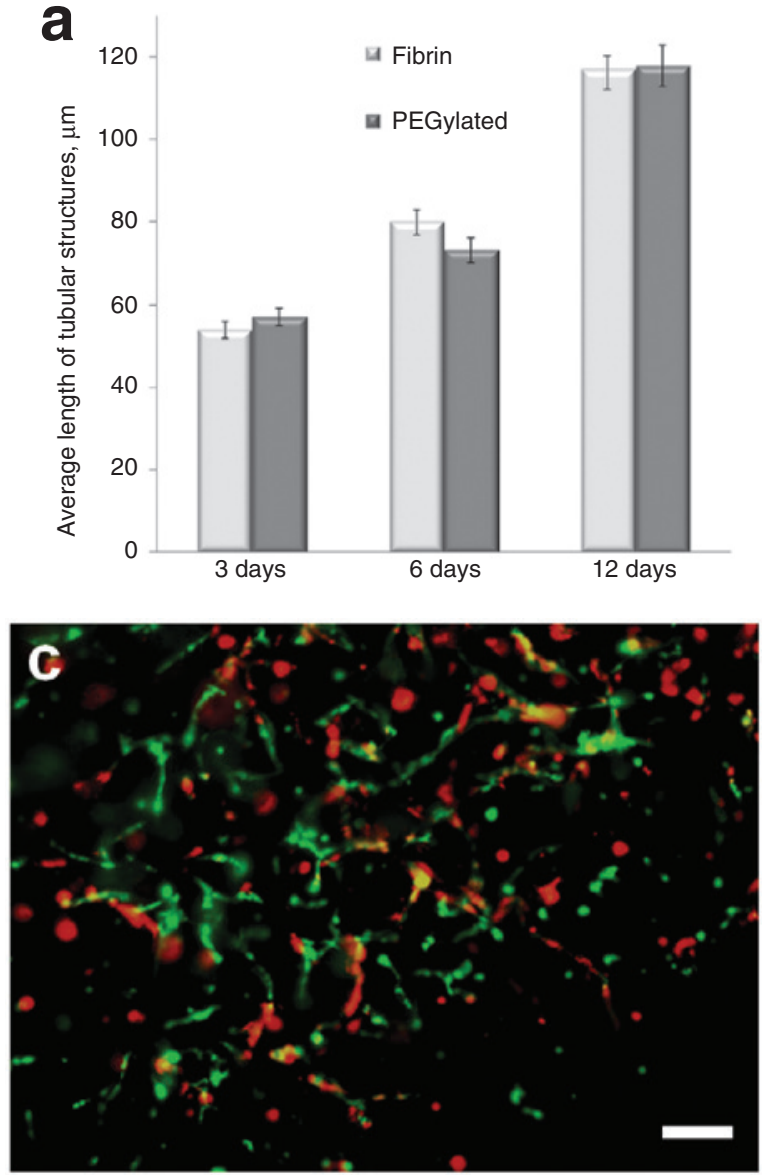
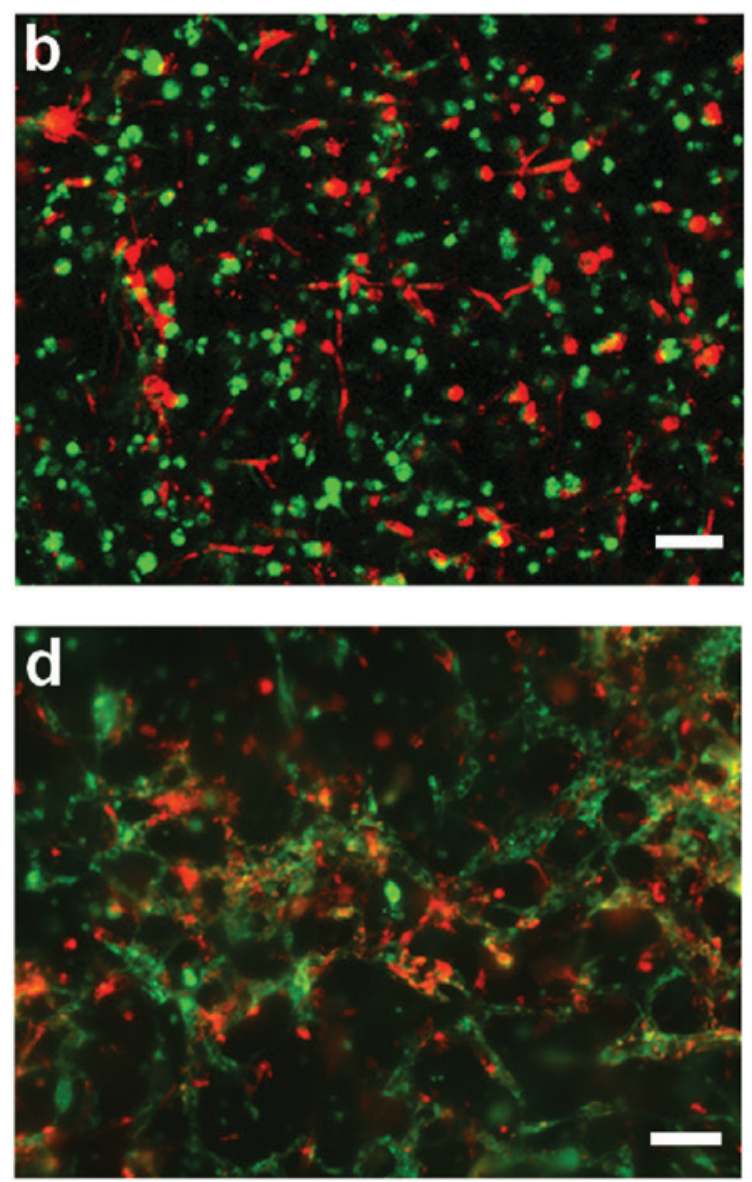

Figure 6: HUVEC and hASC morphological development over 12 days in perfused 5:1 PEGylated fibrin gel cross-linked with $0.2 \mathrm{U}$ thrombin. (a) average length of tubular structures at day 3, 6 and 12; (b) cell morphology within perfused gel at day 2; (c) formation of tubular outgrowths at day 6; and (d) fully interconnected tubular network formed in perfused hydrogel at day 12 . The cells were labeled with DiO (green HUVECs) and DiL (red hASCs) cell trackers prior to the experiment. Scale bars $=100 \mu \mathrm{m}$ long.

in the formation of longer cellular outgrowths and the establishment of cell-to-cell contacts between HUVECs and hASCs (Figure 6c). It was concluded that tubular 3-D network formation by HUVECs was dependent on the co-culture with hASCs, since HUVECs encapsulated in PEGylated fibrin alone failed to establish interconnected tubular networks. It was noted that hASCs can better withstand the dense PEGylated fibrin microenvironment, penetrating the pores of the hydrogel with their extensions much more easily than HUVECs. These cellular paths initiated by hASC spreading can further support the outgrowth of HUVECs (Figure 6d). Confocal microscopy of perfused PEGylated hydrogels after 12 days in culture confirms that HUVEC tube formation is supported by hASCs (Figure 7a, b). Moreover, the images of the tubes show the presence of a continuous lumen enclosed by HUVECs, indicating the formation of microvascular cord within PEGylated fibrin 3D gel (Figure 7c, d).

\section{Discussion}

Main factors by which microenvironmental cues of synthetically modified fibrin gels can impact 3-D cell functions were investigated in this study. When cells are encapsulated in a 3-D ECM-like protein hydrogel, structural features such as fiber size, fiber interconnectivity, and gel density influence cell shape and cytoskeletal architecture. Mechanical forces between cells and their ECM surroundings provide an important set of signals that control cell shape and function [23]. Given the structural diversity of extracellular environment components, it is not surprising that cellular adhesions in 3-D are highly variable. A PEGylation procedure was investigated to control the stability of fibrin gels for long term in vitro culture. By proper conjugation of PEG with a polypeptide bone of a protein molecule, modification of the molecule could be obtained while the biological function (e.g. enzymatic activity or receptor recognition) remains intact [24]. 


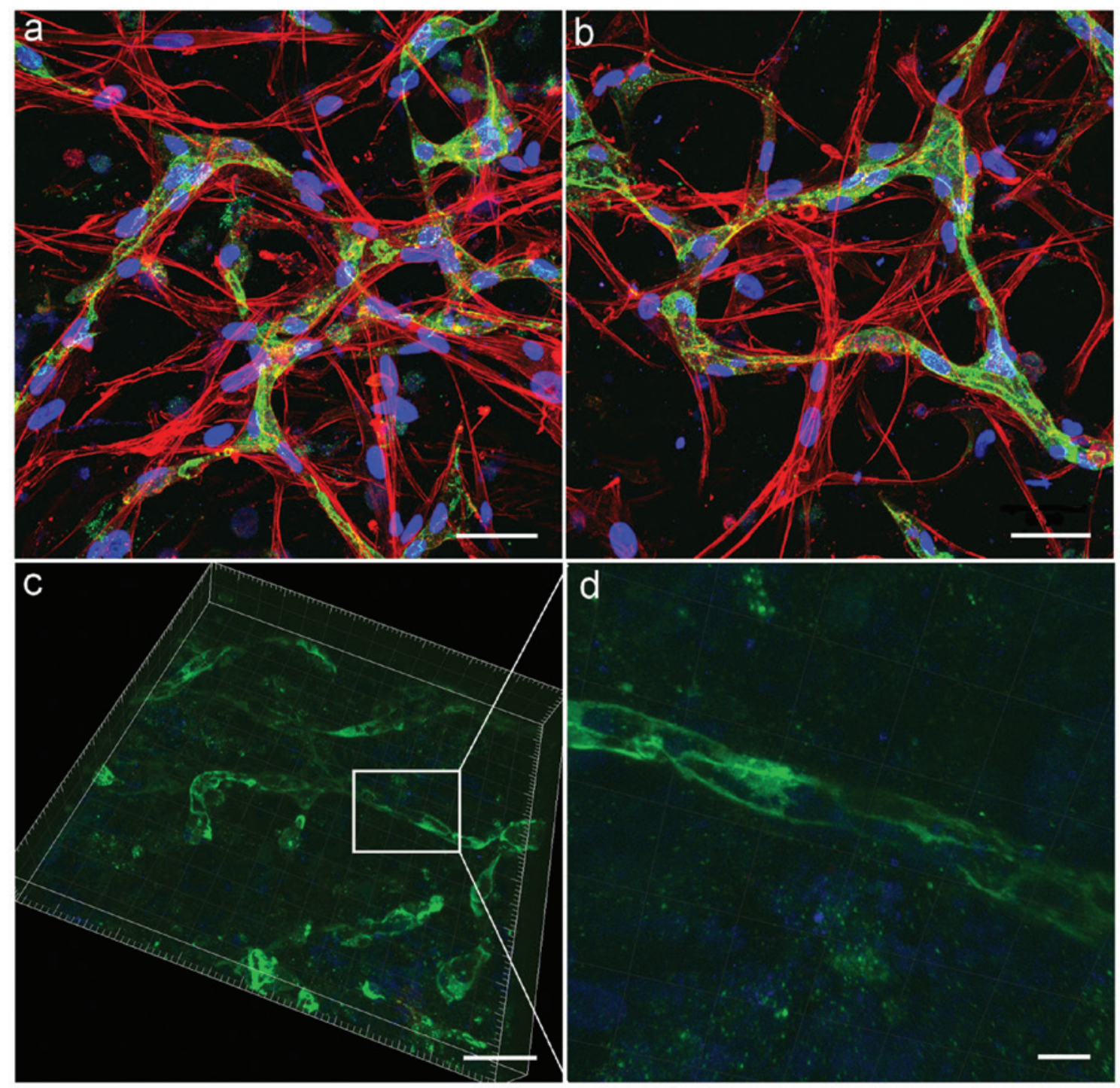

Figure 7: Confocal microscopy images of HUVECs and hASCs co-culture within the perfused PEGylated fibrin construct. (a, b) green HUVECS (CD31 staining), red $\alpha$ SMA staining represents hASCs; (c) 3D representation of confocal image stack showing the formation of HUVECs vascular network and the presence of lumen established by HUVECs (CD31 staining); and (d) enlarged view of HUVEC formed tube. Scale bars $a, b, c=50 \mu \mathrm{m}, \mathrm{d}=10 \mu \mathrm{m}$.

PEGylation of fibrinogen with NHS-functionalized PEG (PEG-NHS) did not influence its ability to undergo enzymatic crosslinking upon reaction with thrombin. Covalent binding of $\mathrm{N}$-succinimidyl functional groups with primary amine groups of fibrinogen molecules was demonstrated using FT-IR analysis (Figure 1). Fibrin, PEGylated in different molar ratios, was used for encapsulation of HUVECs and hASCs in order to evaluate the optimal gel compositions for cell growth, including the ratio of thrombin and fibrinogen. Based on previous scientific reports, we have chosen 10:1 and 5:1 PEGylation ratios for fibrinogen modification $[14,25]$. In contrast to pure fibrin, PEGylated fibrin gels remained fully transparent upon crosslinking with thrombin, which represents a physico-optical benefit and enables deep microscopic analysis of cells cultured within the fibrin hydrogel.

Mechanical and microstructural properties of the PEGylated fibrin hydrogels were elucidated using rheological measurements and SEM observations of the dried gel microarchitecture. In general, PEGylation increased the stiffness of the fibrin gel by approximately $30 \%$; gels prepared with the lower $0.2 \mathrm{U}$ thrombin concentration exhibited slightly lower $G^{*}$ values in comparison to hydrogels prepared with the $1 \mathrm{U}$ thrombin concentration solutions (Figure 1B). It should be noted that mechanical characterizations were performed on bulk hydrogels and may differ 
significantly from what is recognized locally by a single cell [26].

The trends in hydrogel stiffness can be correlated with cellular behavior within PEGylated fibrin hydrogels and with SEM observations of matrix characteristics, confirming strong morphological modification of the fibrillar network by PEG molecules (Figure 1C, D). The fibrillar nature of fibrin is responsible for modulating cell adhesion and mechanical signaling. The nanoscale character of the fibrin fibers is an important feature by which 3-D culture can impact cell function. HUVECs and hASCs cultured in pure, non-modified fibrin gels could relatively quickly develop cell extensions and establish cell-to-cell contacts, while continuing to proliferate, migrating and degrading the fibrin matrix. The 10:1 PEGylated fibrin, however, was determined to be not supportive for 3-D growth of this cell co-culture (Figure 1A, B). It could be observed from SEM images that 10:1 PEGylated fibrin gels represent a very dense-packed bulk architecture, which seems to inhibit cellular spatial distribution, growth, and migration (Figure 1C). In a fibrin gel, PEGylated at 5:1 ratio, HUVECs and hASCs were minimally spread when entrapped into gels prepared using $1 \mathrm{U}$ thrombin. A further decrease in the enzyme concentration resulted in gels with lower stiffnesses, which were able to support 3-D growth of both cell types (Figure 1D).

Fibrin is one of the barriers that angiogenic endothelial cells must penetrate during new blood vessel formation. Invasion and tubulogenesis only takes place if a full-length membrane-associated form of type-1 matrix metalloproteinase (MT1-MMP) is expressed [27]. Recently published analyses revealed a combinatorial MT1-MMP proteolytic program that governs endothelial cell chemotaxis, motility, and adhesion, which ultimately leads to angiogenesis [28]. The potent pericellular fibrinolysin MT1-MMP enables, otherwise surface-dwelling, endothelial cells to penetrate fibrin gels and form tubular structures. In this study, the upregulation of MT1-MMP was noted only in hASC- HUVEC co-cultures, but not in HUVECs and hASCs cultured alone in PEGylated fibrin gels (Figure 3A). These observations additionally prove the dependence of the endothelial cell-mediated vasculogenesis process on co-culture with stromal cells (hASCs). Other endothelial cell MMPs are required for neovascularization of fibrin to occur in vitro and in vivo. MT3-MMP is also involved in the regulation of tube formation by endothelial cells [29]. RT-PCR analysis of MT1-MMP and MT3-MMP genes confirms their upregulation in HUVECshASCs co-cultured in 3-D PEGylated fibrin gels. MMP-2, MMP-9 and MMP-19 have been reported to be implicated in angiogenesis [21, 30, 31]. RT-PCR analysis of their upregulation and downregulation revealed that only gelatinase MMP-2 was constantly active, whereas MMP-9 and MMP-19 genes were active in the first days of perfused culture, but silenced at day 12, when tubular network was formed (Figure 4B). Since MT1-MMP proteolyzes the pro-MMP-2 to its active form that can activate MMP-9, these three MMPs would form a collaborative network to drive the vasculogenic response [27]. The data presented here shows that PEGylated fibrin matrix supports the vasculogenic proteolytic program of HUVEChASC co-culture.

It has been previously demonstrated that co-cultures of hASCs and ECs in 2-D and on Matrigel ${ }^{\circledR}$ in a ratio of 1:3 and 1:7 (ASCs:ECs) produced a stable vascular network with inner layers formed by ECs and outer layers formed by ASCs. This phenomenon was associated with an increased expression of ECM proteins such as laminin, collagen IV, as well as cellular $\alpha$ SMA [2, 4]. In our study, we applied a 1:4 co-culture ratio of hASCs to HUVECs for encapsulation into PEGylated fibrin hydrogel constructs and confirmed that the considerable expression of collagen IV, laminin and $\alpha \mathrm{SMA}$ is dependent on co-culture of hASCs and HUVECs within the PEGylated fibrin gel (Figure 2). Analysis of co-localization of HUVECs (stained for CD31) with hASCs (stained for $\alpha$ SMA) at day 12 indicated that hASCs were localized in the vicinity of HUVEC tubes (Figure 7).

Several studies reported on the formation of microvascular channels by seeding endothelial cells into channels formed within a collagen I gel [32, 33]. There are a number of studies that utilized fibrin gel for the creation of micro vessel tubes [34-36]. The proof of principle described in this work utilized a similar approach for the formation of a single fluidic channel within the cell laden PEGylated fibrin gel. Here we first focused on the evaluation of optimum gel properties for induction of a cellular lumenalized network by co-culture of vasculogenic cells. However, the principle of a sacrificial material interconnected capillary network formation based on utilization of gelatin [37] or sugar [35] is certainly applicable to the approach described in this paper. Using a combination of photolithography and soft lithography methods, it should be possible to fabricate complex interconnected microchannel networks from a sacrificial material and apply it for embedding within cell laden PEGylated fibrin gels. By liquefying and flushing away the sacrificial material, the resulting branched micro-channels can represent a capillary network for conducting vasculogenic perfusion studies. Subsequent seeding of these channels with endothelial cells will further increase the similarity of the channel with living tissue. This approach represents a 
versatile tool for investigation of vasculogenesis, anastomosis, and other basic and applied topics related to vascular biology, including drug testing and cancer research. Using this approach, perfusable 3-D microfluidic capillary networks with various dimensions can be formed within the cell-containing hydrogel in vitro. Using a multichannel peristaltic pump, hydrogel perfusion experiments could be performed in a parallel manner, examining up to 6 devices at the same time. Due to the relative optical transparency of PEGylated fibrin gels and utilization of transparent PDMS as microfluidic chamber, this approach is compatible with live-cell microscopy for monitoring of cellular response.

\section{Conclusions}

This proof of principle study demonstrates the use of hydrogel-based microfluidic system for investigations of vasculogenesis within perfused synthetically-modified fibrin gels. Mechanical properties of PEGylated hydrogel compositions were evaluated by rheological measurements and compared with rheological characteristics of pure fibrin gel. The optimal hydrogel compositions considering the degree of synthetic modification and thrombin content, as well as, optimal cellular density and co-culture ratio of HUVECs and hASCs have been evaluated by investigations of the morphological development of cells within the 3-D culture with fluorescence microscopy. It has been demonstrated that cells cultured within PEGylated gel do not lose their protheolithic program and express certain variety of MMPs, which are responsible for the formation of tubular network. Upon further evaluation of appropriate mechanical and chemical microenvironmental qualities, the PEGylated fibrin microfluidic approach can be used for incorporating other cell types of interest, thus representing a unique experimental platform for tissue engineering.

Acknowledgments: The authors would like to acknowledge Dr. Daria Guseva (MHH) for the assistance in confocal microscopy imaging. Anastasia Shpichka thanks the DAAD and the Ministry of Education and Science of the Russian Federation (Michail Lomonosov program) for the research scholarship. This work was supported by the DFG Cluster of Excellence REBIRTH and Low Saxony project Biofabrication for Nife. Grant of the Government of the Russian Federation for the Support of Scientific Investigations under the Supervision of Leading Scientists Contract No. 14.B25.31.0019 is also acknowledged.

\section{References}

1. Koike N, Fukumura D, Gralla O, Au P, Schechner JS, Jain RK. Creation of long-lasting blood vessels. Nature. 2004;428:138-9.

2. Merfeld-Clauss S, Gollahalli N, March KL, Traktuev DO. Adipose tissue progenitor cells directly interact with endothelial cells to induce vascular network formation. Tissue Eng Part A. 2010;16:2953-66.

3. Montano I, Schiestl C, Schneider J, Pontiggia L, Luginbühl J, Biedermann T, et al. Formation of human capillaries in vitro: the engineering of prevascularized matrices. Tissue Eng Part A. 2010;16:269-82.

4. Traktuev DO, Prater DN, Merfeld-Clauss S, Sanjeevaiah AR, Saadatzadeh MR, Murphy M, et al. Robust functional vascular network formation in vivo by cooperation of adipose progenitor and endothelial cells. Cir Res. 2009;104:1410-20.

5. Auerbach R, Auerbach W. Vasculogenesis and angiogenesis. In: Fan T, Kohn E, editors. The new angiotherapy. Totowa, N): Humana Press Inc.; 2002, pp. 1-6.

6. Prewitz MC, Seib FP, von Bonin M, Friedrichs J, Stißel A, Niehage $C$, et al. Tightly anchored tissue-mimetic matrices as instructive stem cell microenvironments. Nat Methods. 2013;10:788-94.

7. Hughes CS, Postovit LM, Lajoie GA. Matrigel: a complex protein mixture required for optimal growth of cell culture. Proteomics. 2010;10:1886-90.

8. Auerbach R, Lewis R, Shinners B, Kubai L, Akhtar N. Angiogenesis assays: a critical overview. Clin Chem. 2003;49:32-40.

9. Shaikh FM, Callanan A, Kavanagh EG, Burke PE, Grace PA, McGloughlin TM. Fibrin: a natural biodegradable scaffold in vascular tissue engineering. Cells Tissues Organs. 2008;188:333-46.

10. Rowe SL, Lee S, Stegemann JP. Influence of thrombin concentration on the mechanical and morphological properties of cell-seeded fibrin hydrogels. Acta Biomater. 2007;3:59-67.

11. Bensaïd W, Triffittb JT, Blanchata C, Oudinaa K, Sedela L, Petite H. A biodegradable fibrin scaffold for mesenchymal stem cell transplantation. Biomaterials. 2003;24:2497-502.

12. Bryant SJ, Durand KL, Anseth KS. Manipulations in hydrogel chemistry control photoencapsulated chondrocyte behavior and their extracellular matrix production. J Biomed Mater Res A. 2003;67:1430-6.

13. Dikovsky D, Bianco-Peled H, Seliktar D. The effect of structural alterations of PEG-fibrinogen hydrogel scaffolds on 3-D cellular morphology and cellular migration. Biomaterials. 2006;27:1496-506.

14. Galler KM, Cavender AC, Koeklue U, Suggs LJ, Schmalz G, D'Souza RN. Bioengineering of dental stem cells in a PEGylated fibrin gel. Regen Med. 2011;6:191-200.

15. Zhang G, Drinnan CT, Geuss LR, Suggs LJ. Vascular differentiation of bone marrow stem cells is directed by a tunable threedimensional matrix. Acta Biomater. 2010b;6:3395-403.

16. Zhang G, Wang X, Wang Z, Zhang J, Suggs L. A PEGylated fibrin patch for mesenchymal stem cell delivery. Tissue Eng. 2006;12:9-19.

17. Gruene M, Pflaum M, Deiwick A, Koch L, Schlie S, Unger C, et al. Adipogenic differentiation of laser-printed 3D tissue grafts consisting of human adipose-derived stem cells. Biofabrication. 2011;3:015005. 
18. Hesse M, Meier H, Zeeh B. Spectroscopic methods in organic chemistry. Stuttgart: Georg Thieme Verlag; 1997.

19. Touahir L, Chazalviel J-N, Sam S, Moraillon A, Henry de Villeneuve $C$, Allongue $P$, et al. Kinetics of activation of carboxyls to succinimidyl ester groups in monolayers grafted on silicon: an in situ real-time infrared spectroscopy study. J Phys Chem C. 2011;115:6782-87.

20. Frey BL, Corn RM. Covalent attachment and derivatization of poly(L-lysine) monolayers on gold surfaces as characterized by polarization-modulation FT-IR spectroscopy. Anal Chem. 1996;68:3187-93.

21. Taraboletti G, D’Ascenzo S, Borsotti P, Giavazzi R, Pavan A, Dolo V. Shedding of the matrix metalloproteinases MMP-2, MMP-9, and MT1-MMP as membrane vesicle-associated components by endothelial cells. Am J Pathol. 2002;160:673-80.

22. Lafleur MA, Handsley MM, Knäuper V, Murphy G, Edwards DR. Endothelial tubulogenesis within fibrin gels specifically requires the activity of membrane-type-matrix metalloproteinases (MT-MMPs). J Cell Sci. 2002;115:3427-38.

23. Hoffman BD, Grashoff C, Schwartz MA. Dynamic molecular processes mediate cellular mechanotransduction. Nature. 2011;475:316-23.

24. Veronese FM. Peptide and protein PEGylation: a review of problems and solutions. Biomaterials. 2001;22:405-17.

25. Zhang G, Drinnan CT, Geuss RA, Suggs LJ. Vascular differentiation of bone marrow stem cells is directed by a tunable threedimensional matrix. Acta Biomater. 2010a;6:3395-3403.

26. Tranqui L, Tracqui P. Mechanical signalling and angiogenesis. The integration of cell-extracellular matrix couplings. Comptes Rendus de l'Académie des Sciences-Series III-Sciences de la Vie. 2000;323:31-47.

27. Chun T-H, Sabeh F, Ota I, Murphy H, McDonagh KT, Holmbeck K. MT1-MMP-dependent neovessel formation within the confines of the three-dimensional extracellular matrix. J Cell Biol. 2004;167:757-67.
28. Koziol A, Gonzalo P, Mota A, Pollán Á, Lorenzo C, Colomé N, et al. The protease MT1-MMP drives a combinatorial proteolytic program in activated endothelial cells. FASEB J. 2012;26: 4481-94.

29. Plaisier M, Kapiteijn K, Koolwijk P, Fijten C, Hanemaaijer R, Grimbergen JM, et al. Involvement of membrane-type matrix metalloproteinases (MT-MMPs) in capillary tube formation by human endometrial microvascular endothelial cells: role of MT3MMP. J Clin Endocrinol Metab. 2004;89:5828-36.

30. Sternlicht MD, Werb Z. How matrix metalloproteinases regulate cell behaviour. Annu Rev Cell Dev Biol. 2001;17:463-516.

31. Titz B, Dietrich S, Sadowski T, Beck C, Petersen A, Sedlacek R. Activity of MMP-19 inhibits capillary-like formation due to processing of nidogen-1. Cell Mol Life Sci. 2004;61:1826-33.

32. Chrobak KM, Potter DR, Tien J. Formation of perfused, functional microvascular tubes in vitro. Microvasc Res. 2006;71:185-96.

33. Price GM, Wong KH, Truslow JG, Leung AD, Acharya C, Tien J. Effect of mechanical factors on the function of engineered human blood microvessels in microfluidic collagen gels. Biomaterials. 2010;31:6182-9.

34. Lesman A, Koffler J, Atlas R, Blinder YJ, Kam Z, Levenberg S, et al. Engineering vessel-like networks within multicellular fibrin-based constructs. Biomaterials. 2011;32:7856-69.

35. Miller JS, Stevens KR, Yang MT, Baker BM, Nguyen DT, Cohen DM, et al. Rapid casting of patterned vascular networks for perfusable engineered three-dimensional tissues. Nat Mater. 2012;11:768-74.

36. Shamloo A, Heilshorn SC. Matrix density mediates polarization and lumen formation of endothelial sprouts in VEGF gradients. Lab Chip. 2010;10:3061-8.

37. Lee W, Lee V, Polio S, Keegan P, Lee JH, Fischer K, et al. Ondemand three-dimensional freeform fabrication of multi-layered hydrogel scaffold with fluidic channels. Biotechnol Bioeng. 2010;105:1178-86. 